\title{
Probabilistic sensitivity of Nash equilibria in multi-agent games: a wait-and-judge approach
}

\author{
Filiberto Fele and Kostas Margellos
}

\begin{abstract}
Motivated by electric vehicle charging control problems, we consider multi-agent noncooperative games where, following a data driven paradigm, unmodeled externalities acting on the players' objective functions are represented by means of scenarios. Building upon recent developments in scenario-based optimization, based on the evaluation of the computed solution, we accompany the Nash equilibria of the uncertain game with an a posteriori probabilistic robustness certificate, providing confidence on the probability that the computed solution remains unaffected when a new uncertainty realisation is encountered. The latter constitutes, to the best of our knowledge, the first application of the so-called scenario approach to multi-agent Nash equilibrium problems. The efficacy of our approach is demonstrated in simulation for the charging coordination of an electric vehicle fleet.
\end{abstract}

\section{INTRODUCTION}

The role of game theory in the management and control of cyberphysical systems [1] is now consolidated, counting on a significant research effort in the last decades [2]. The significance of the concept of Nash equilibrium (NE) in this context comes from the ability to predict the resolution of conflicts between selfish, rational agents [3]. NE problem formulations have become popular candidates for distributed and decentralized control architectures, as they naturally lend themselves to price-based implementations, easily fitting in the operational requirements of liberalized resource markets [4]. Applications in smart grid, transportation, and IT are envisioned among the possible beneficiaries [5], [6], [7], [8], [9]. In connection with public infrastructures, a large number of studies followed the seminal work of Wardrop [10], focusing on the relation between the achieved equilibria and the social welfare; among recent results, we mention [11], [12], [7], [13], [14].

According to the definition of NE [15], the aforementioned studies address deterministic settings where the systems conditions (e.g., prices in an incentive scheme) are assumed to be known in advance. Such an assumption is not reflected in realistic scenarios, where the relevant information may be subject to significant uncertainties: this concept becomes paramount in the framework of cyberphysical systems, where the heterogeneity and complexity of the interactions between the constituting parts heavily hinders the task of predicting the system behaviour [16], [17], [18]. Indeed, since the complete knowledge of the game was questioned by [19], uncertainty has been widely addressed in noncooperative games, by adopting stochastic or robust (worst-case) approaches. In the first case, both chance-constrained (riskaverse) [20], [21] or expected payoff criteria [22], [23], [24],

Funding from the UK Engineering and Physical Sciences Research Council (EPSRC) under grant agreement EP/P03277X/1 is gratefully acknowledged.

The authors are with the Department of Engineering Science, University of Oxford, OX1 3PJ, UK ffiliberto.fele, kostas.margellos\} deng.ox.ac.uk
[25], [26] have been considered. Due to their approach, these studies pivot around given hypotheses on the underlying probability distribution of the uncertainty. In the second case, results build upon robust control theory [3], [27], and as such they hold for given characterizations of the uncertainty set. We refer the reader to [28], [16] for an extended literature review.

We depart by previous robust game-theoretical literature by characterizing uncertainty on the objective function through a data-driven methodology. In particular, our results are founded on recent developments in the scenario optimization [29]. In this framework, uncertainty is characterized by a finite set of scenarios [30] obtainable, e.g., from historical data [31]. One essential feature of the proposed approach is to allow the definition of probabilistic performance certificates for the solution-without requiring any additional knowledge on the uncertainty except for the available set of scenarios used in the solution computation [32]. Notably, the results presented in [33] constitute a breakthrough in this sense: under mild convexity assumptions, these provide tight a priori bounds on the probability of constraint violation of a scenario program solution. However, in the present context, these results can be quite conservative in general, as they depend on the dimension of the decision space: in the considered setting, a considerable overhead is due to the size of the scenario set. Moreover, in multi-agent games, this translates into a number of decision variables increasing with the number of players. Nonetheless, the theory presented in [29] allows to circumvent this problem by means of an $a$ posteriori analysis of the solution: in this case, the only determining factor in the robustness performance is the cardinality of the subset of samples constraining the solution.

In this paper, we contribute to the state-of-the-art of NE problem solution algorithms in the following aspects:

- We address the robustness of strategic equilibria in multi-agent settings, characterized as NEs of noncooperative minmax games. We propose a partially-cooperative approach to achieve robustness to uncertainty globally affecting the players' objective functions. We note that although this approach shares theoretical grounds with [34], we depart from it by admitting the presence of multiple maximisers. Besides expanding the scope of the proposed game model, this is pivotal to the application of the adopted scenario-based framework.

- We propose a decentralized solution approach: relying on the action of a coordinator, no communication between the agents is contemplated, thus relaxing the communicational requirements of related results (e.g., [25]). To circumvent the nondifferentiabilty of the game, hindering the application of conventional decentralised techniques, we follow the methodology in [35] and resort to an augmented game. Major advantages of this approach are $(i)$ the solution 
computation enjoys the same convergence properties of stateof-the-art decentralized algorithms for monotone games [8], and $(i i)$ the uncertain component of the objective function can fall within the broader class of weakly convex functions [35]. We note that, as an alternative, the minmax problem can be cast into the class of generalized Nash games by an epigraph reformulation: however, decentralized solution algorithms for this class of games impose generally restrictive assumptions, e.g., affine coupling constraints [9], [7], [11], which do not necessarily fit the format of the resulting epigraphic constraint.

- We adopt a data-driven paradigm and represent the unmodelled uncertainty by means of scenarios [30]. We then accompany the solution with prescribed robustness levels, while dropping the standard requirements on the knowledge of the uncertainty [29]. To the best of our knowledge, this work sets the first step in the formalization of scenariobased robustness in noncooperative games. In doing this, we overcome significant limitations of stochastic approaches, namely the computational cost of Monte Carlo simulations, and the absence of guarantees for the solution.

The rest of the paper is structured as follows. In Section II we introduce a motivating example, and then provide a more general formulation of the addressed noncooperative minmax problem. We present the main result of the paper in Section III. Section IV introduces necessary ingredients for the proof, described in Section $\mathrm{V}$ along with a methodology for the enumeration of the uncertainty samples supporting the NE. Finally, numerical results are illustrated on an electricvehicle charging coordination problem.

\section{PRoblem STATEMENT}

\section{A. Motivating example: coordinated EV charging}

Let the set $\mathcal{N}=\{1, \ldots, N\}$ designate the finite population of EV agents. The demand profile of the EVs (henceforth the strategy) $x_{i} \in \mathbb{R}_{+}^{q}$ of agent $i \in \mathcal{N}$ must fulfil individual constraints described by the set $\mathcal{X}_{i} \subset \mathbb{R}^{q}$. We denote by $x=\left(x_{i}\right)_{i \in \mathcal{N}} \in \mathcal{X} \subset \mathbb{R}^{n}$, with $n=q N$, the collection of strategies relative to all the agents, where $\mathcal{X}=\mathcal{X}_{1} \times \cdots \times \mathcal{X}_{N}$.

Each agent determines its strategy as a response to a pricing signal received from a coordinator, and synthesized as a function of the global strategy $x$; such a signal can represent, for instance, the variable unit cost of a consumed resource. The possible influence of uncertainty (e.g., externalities acting on the energy spot market) on the price is modelled by the parameter $\theta \in \Theta$. In particular, we assume that a nominal and an uncertain component can be distinguished: thus, given $x$ and $\theta$, the utility of agent $i \in \mathcal{N}$ is

$$
U_{i}(x, \theta)=-\left(x_{i}^{\top} p_{0}(x)+x_{i}^{\top} p_{\theta}(x, \theta)\right),
$$

where $p_{0}$ and $p_{\theta}$ respectively represent the nominal and the uncertain component of the price.

\section{B. Noncooperative minmax game}

To cope with the uncertain component of the price, we consider the minimisation of its average impact on the value of $U_{i}$, for all $i \in \mathcal{N}$. In other words, each agent $i \in \mathcal{N}$ computes

$$
x_{i}^{*}=\underset{x_{i} \in \mathcal{X}_{i}}{\arg \min } J_{i}\left(x_{i}, x_{-i}\right)
$$

where $J_{i}: \mathbb{R}^{n} \rightarrow \mathbb{R}$ is defined as

$$
\begin{aligned}
J_{i}\left(x_{i}, x_{-i}\right) \triangleq x_{i}^{\top} p_{0}\left(x_{i}, x_{-i}\right) & \\
& +\max _{\theta \in \Theta} \frac{1}{N} \sum_{j \in \mathcal{N}} x_{j}^{\top} p_{\theta}\left(x_{i}, x_{-i}, \theta\right) .
\end{aligned}
$$

We can write (2) in a more general form as

$$
J_{i}\left(x_{i}, x_{-i}\right)=f_{i}\left(x_{i}, x_{-i}\right)+w\left(x_{i}, x_{-i}\right),
$$

where $f_{i}(x)$ expresses local objectives as a function of local and (possibly) global strategies whereas, for some common objective $g(x, \theta), w(x)=\max _{\theta \in \Theta} g(x, \theta)$ evaluates the worst-case realization over the uncertainty set $\Theta$. As a consequence, the solution of (1) entails a certain level of cooperation between the agents, which can be facilitated in settings where their interests are (at least to some degree) aligned (e.g., electric vehicles participating in the same aggregation plan, or belonging to a centrally managed fleet).

The setting described above is modelled by a noncooperative minmax game, defined by the tuple $\mathcal{G}=$ $\left\langle\mathcal{N},\left(\mathcal{X}_{i}\right)_{i \in \mathcal{N}},\left(J_{i}\right)_{i \in \mathcal{N}}, \Theta\right\rangle$, where $\mathcal{N}$ is the set of players, $\mathcal{X}_{i}, J_{i}$ are respectively the strategy set and the cost function for each player $i \in \mathcal{N}$, and $\Theta$ is the uncertainty set.

We consider the following blanket assumptions:

Assumption 1: For every fixed $x_{-i} \in \mathcal{X}_{-i}$ and $\theta \in \Theta$, the function $f_{i}\left(\cdot, x_{-i}\right)+g\left(\cdot, x_{-i}, \theta\right)$ is convex and continuously differentiable for all $i \in \mathcal{N}$. Furthermore, the local constraint set $\mathcal{X}_{i}$ is nonempty, compact and convex for all $i \in \mathcal{N}$, and $\Theta \subset \mathbb{R}^{d}$ is bounded.

Assumption 2: Let $\mathcal{D}_{x} \subseteq \mathbb{R}^{n}$ be an open convex set such that $\mathcal{X} \subset \mathcal{D}_{x}$. The function $g$ is twice differentiable on $\mathcal{D}_{x}$ and, for each $i \in \mathcal{N}, f_{i}$ is twice differentiable on $\mathcal{D}_{x}$.

We note that for $f_{i}\left(\cdot, x_{-i}\right)+g\left(\cdot, x_{-i}, \theta\right)$ to be convex it is sufficient that $f_{i}\left(\cdot, x_{-i}\right)$ is weakly convex on $\mathcal{X}_{i}$ and $g(\cdot, \theta)$ is weakly convex on $\mathcal{X}$, with constant $\chi^{f}$ and $\chi^{g}$ respectively, such that $\chi^{f}+\chi^{g} \geq 0$. For a definition of weak convexity see (13)-(14).

\section{ROBUST NONCOOPERATIVE GAME}

The solution of the robust game $\mathcal{G}$ requires each agent to solve a minmax optimisation problem. Even in cases where this is numerically tractable (for example when the uncertainty of the value of $g(\cdot, \theta)$ is well-behaved), the attainment of a robust solution still requires the full characterisation of the set $\Theta$.

\section{A. Scenario-based approach}

Here we suppose instead that, however large, the available set of data (e.g., historical data) only allows to achieve a partial characterisation of the uncertainty. Following a databased approach, it is still possible to consider a "sampled" version of the problem, using a finite set of uncertainty realizations $\theta_{1}, \cdots, \theta_{M} \in \Theta$. This results in the finite $\operatorname{minmax}$ game $\widetilde{\mathcal{G}}=\left\langle\mathcal{N},\left(\mathcal{X}_{i}\right)_{i \in \mathcal{N}},\left(\widetilde{J}_{i}\right)_{i \in \mathcal{N}},\left\{\theta_{j}\right\}_{j=1}^{M}\right\rangle$, where

$$
\widetilde{J}_{i}\left(x_{i}, x_{-i}\right)=f_{i}\left(x_{i}, x_{-i}\right)+\tilde{w}\left(x_{i}, x_{-i}\right),
$$

and $\tilde{w}(x)=\max _{m \in\{1, \cdots, M\}} g\left(x, \theta_{m}\right)$ approximates $w(x)$ in (3). We consider the following solution concept for $\widetilde{\mathcal{G}}$ : 
Definition 3 (Nash equilibrium): Let $\Omega \subseteq \mathcal{X}$ denote the set of Nash equilibria of $\widetilde{\mathcal{G}}$, defined as

$$
\begin{aligned}
\Omega \triangleq\left\{\bar{x}=\left(\bar{x}_{i}\right)_{i \in \mathcal{N}}\right. & \in \mathcal{X}: \\
\bar{x}_{i} & \left.\in \underset{x_{i} \in \mathcal{X}_{i}}{\arg \min } \widetilde{J}_{i}\left(x_{i}, \bar{x}_{-i}\right), \forall i \in \mathcal{N}\right\} .
\end{aligned}
$$

We point out that $\tilde{w}$ is a random variable, subject to the particular extraction of $M$ elements from $\Theta$. As a consequence, the composition of the set $\Omega$ is subject to the given multiextraction $\left(\theta_{1}, \ldots, \theta_{M}\right) \in \Theta^{M}$. For notational simplicity, we will not make this dependence explicit in the rest of the document.

\section{B. A posteriori robustness certification}

A question that naturally arises is how robust a solution $x^{*} \in \Omega$ is against unknown scenarios-i.e., scenarios not included in the available dataset, and how such a solution relates to the equilibria of the original game $\mathcal{G}$. In the remainder of this section we show that a formal answer to this question can be provided through a sensitivity analysis of the solution over the set of samples $\left\{\theta_{1}, \ldots, \theta_{M}\right\}$ used for its derivation. Most importantly, this estimate can be performed without requiring any further knowledge of the uncertainty except for the aforementioned $M$ samples. The main resultrelying on the developments in scenario-based optimization recently presented in [29] — is a robustness certificate that quantifies the probability for a $\mathrm{NE}$ of the approximated game $\widetilde{\mathcal{G}}$ to remain an equilibrium when a new scenario of uncertainty is realized. The formal statement of this result relies on the following property:

Assumption 4: The uncertainty set $\Theta$ is endowed with a $\sigma$-algebra $\mathcal{D}$, and a probability $\mathbb{P}$ can be assigned over $\mathcal{D}$.

In order to proceed we provide some basic definitions:

Definition 5 (Support sample [33]): Fix any sample multiextraction $\left(\theta_{1}, \ldots, \theta_{M}\right) \in \widetilde{\Theta}^{M}$. Let $x^{*}$ be a solution of the finite minmax game $\widetilde{\mathcal{G}}$ obtained as a function of the previous uncertainty sample, and let $x^{\circ}$ be the solution obtained by the same algorithm from the reduced set $\left\{\theta_{1}, \ldots, \theta_{s-1}, \theta_{s+1}, \ldots, \theta_{M}\right\}$. The sample $\theta_{s}$ is called a support sample if $x^{\circ} \neq x^{*}$.

In the following, we refer to the support dimension $\bar{n} \leq M$ as the number of samples supporting a given NE $x^{*} \in \Omega$ [36]. Note that $\bar{n}$ is itself a random variable as it depends on the multi-sample $\left\{\theta_{1}, \ldots, \theta_{M}\right\}$.

Given $\theta \in \Theta$, let $\Omega^{+}$designate the NE set of the game $\widetilde{\mathcal{G}}^{+}=\left\langle\mathcal{N},\left(\mathcal{X}_{i}\right)_{i \in \mathcal{N}},\left(\widetilde{J}_{i}\right)_{i \in \mathcal{N}},\left\{\theta_{j}\right\}_{j=1}^{M} \cup\{\theta\}\right\rangle$, defined over the $M+1$ scenarios $\left\{\theta_{1}, \ldots, \theta_{M}, \theta\right\}$. Then, for all $x^{*} \in \Omega$, let $V\left(x^{*}\right) \triangleq \mathbb{P}\left\{\theta \in \Theta: x^{*} \notin \Omega^{+}\right\}$. Finally, following [29], let $\varepsilon:\{0, \ldots, M\} \rightarrow[0,1]$ be a function satisfying

$$
\begin{aligned}
& \varepsilon(M)=1, \\
& \sum_{k=0}^{M-1}\left(\begin{array}{c}
M \\
k
\end{array}\right)(1-\varepsilon(k))^{M-k}=\beta,
\end{aligned}
$$

for any fixed $\beta \in(0,1)$. We can now state our main result:

Theorem 6: Fix $\beta \in(0,1)$ and let $\varepsilon(\cdot)$ be defined as in (6). Under Assumptions 1, 2 and 4 the following holds:

(i) There exists a single-valued decentralized mapping $\widetilde{\Phi}: \Theta^{M} \rightarrow \Omega$ from the set of feasible strategies to the set of equilibria of $\widetilde{\mathcal{G}}$; (ii) Let $\bar{n}$ be the support dimension of $x^{*}=\widetilde{\Phi}\left(\theta_{1}, \ldots, \theta_{M}\right)$, with $\left\{\theta_{m}\right\}_{m=1}^{M}$ being independent random samples from $\Theta$. Then

$$
\mathbb{P}^{M}\left\{\left(\theta_{1}, \ldots, \theta_{M}\right) \in \Theta^{M}: V\left(x^{*}\right)>\varepsilon(\bar{n})\right\} \leq \beta .
$$

Proof: See Section V-A.

According to Theorem 6, any solution $x^{*} \in \Omega$-selected by suitably defining the single-valued mapping $\widetilde{\Phi}$ - can be endowed with probabilistic guarantees on its robustness against uncertainty, given the knowledge of its support dimension $\bar{n}$. A fundamental interpretation of Theorem 6 is that it quantifies, with a given confidence level, the probability that the NE $x^{*}$, computed on the randomly extracted samples $\left(\theta_{1}, \ldots, \theta_{M}\right) \in \Theta^{M}$, remains a solution of the game $\mathcal{G}$ when a new sample $\theta \in \Theta$ is produced.

We point out that a tighter confidence could be obtained by means of the results of [37]; however, this would require the imposition of a non-degeneracy assumption on the problem. ${ }^{1}$ In view of not restricting the class of problems considered, we limit attention to confidence levels in the form of (6).

An a posteriori estimate of the support dimension can be obtained through different methodologies, whose design may be tuned on the specific case. A greedy algorithm of general application, which is a straightforward implementation of Definition 5 , is described in $[29, \S I I]$. In Section V-B we present a more efficient approach that exploits the structure of the considered noncooperative minmax games.

\section{Decentralized NE COMPutation}

In this section we study in more detail the existence of a single-valued mapping $\widetilde{\Phi}$, necessary for the proof of Theorem 6. In particular, we consider the case in which the image of $\widetilde{\Phi}$ corresponds to the limit of a decentralized solution algorithm for the game $\widetilde{\mathcal{G}}$. To address this, we characterise the NEs of $\widetilde{\mathcal{G}}$ as solutions of variational inequalities (VI). Established results in this framework allow to define sufficient conditions for the existence of equilibria, and set the foundations for the design of decentralized solution procedures [8].

\section{A. VI analysis}

At the core of the use of the VI framework for the modelling of noncooperative games solutions is the correspondence between the so-called VI problem, which takes the form (for a given domain $\mathcal{D} \subseteq \mathbb{R}^{d}$ )

$$
\begin{aligned}
& \text { find } z^{*} \in \mathcal{D} \\
& \text { s.t. }\left(z-z^{*}\right)^{\top} F\left(z^{*}\right) \geq 0, \forall z \in \mathcal{D},
\end{aligned}
$$

and the first-order optimality conditions corresponding to a NE (see Definition 3) [39, § 1.5]. This model naturally hinges on the differentiability of the problem at hand; however, we can observe that, due to the max operator, the players' objective functions defining $\widetilde{\mathcal{G}}$ are in general nondifferentiable.

With this in mind, let us define the augmented game $\widehat{\mathcal{G}}$ between $N+1$ players. In $\widehat{\mathcal{G}}$ each agent $i \in \mathcal{N}$, given $x_{-i}$

\footnotetext{
${ }^{1}$ For a definition of degeneracy and of minimal support, we refer the reader to [38], [37].
} 
and $y \equiv\left(y_{m}\right)_{m=1}^{M}$, will compute

$$
x_{i}^{*} \in \underset{x_{i} \in \mathcal{X}_{i}}{\arg \min } f_{i}\left(x_{i}, x_{-i}\right)+\underbrace{\sum_{m=1}^{M} y_{m} g\left(x_{i}, x_{-i}, \theta_{m}\right)}_{\hat{g}\left(x_{i}, x_{-i}, y\right)},
$$

where $\hat{g}(x, y)$ follows from the equivalence

$$
\max _{m \in\{1, \ldots, M\}} g\left(x, \theta_{m}\right)=\max _{y \in \Delta} \sum_{m=1}^{M} y_{m} g\left(x, \theta_{m}\right),
$$

with $\Delta=\left\{y \in \mathbb{R}^{M}: y \geq 0, \sum_{m=1}^{M} y_{m}=1\right\}$ being the simplex in $\mathbb{R}^{M}$ [40, Lemma 6.2.1]. The additional player (the coordinator), given $x$, will act instead as a maximizing player for the uncertain component of $\widetilde{J}_{i}, i \in \mathcal{N}$,

$$
y^{*} \in \underset{y \in \Delta}{\arg \max } \hat{g}(x, y) \text {. }
$$

The following relation holds between $\widetilde{\mathcal{G}}$ and $\widehat{\mathcal{G}}$ :

Lemma 7 (Thm. 1 [35]): Let the pair $\left(x^{*}, y^{*}\right) \in \mathcal{X} \times \Delta$ be a NE of the game $\widehat{\mathcal{G}}$. Then $x^{*}$ is a NE of $\widetilde{\mathcal{G}}$.

Note that $\widehat{\mathcal{G}}$ is differentiable under Assumption 1 . Therefore this fundamental result (holding for all randomly extracted set of uncertainty realizations $\left\{\theta_{1}, \ldots, \theta_{M}\right\} \in \Theta$ ) enables the characterization of the NEs of $\widetilde{\mathcal{G}}$ by means of VIs.

We start by defining the mapping $F(x, y): \mathcal{X} \times \Delta \rightarrow$ $\mathbb{R}^{(n+M)}$ as the pseudo-gradient [39, Sec. 1.4.1]

$$
F(x, y) \triangleq\left[\begin{array}{c}
\left(\nabla_{x_{i}} f_{i}(x)+\nabla_{x_{i}} \hat{g}(x, y)\right)_{i \in \mathcal{N}} \\
-\left(\nabla_{y_{m}} \hat{g}(x, y)\right)_{m=1}^{M}
\end{array}\right] .
$$

By letting $z \triangleq(x, y)$ and $\mathcal{D} \triangleq \mathcal{X} \times \Delta$ we see that (8b) represents the concatenation of the first-order optimality conditions for the $N+1$ individual problems described by (9) and (10). In the following, we refer to the problem described by (8) and (11) as $\operatorname{VI}(F, \mathcal{X} \times \Delta)$.

It turns out that under Assumption 1 any $\mathrm{NE}$ of $\widehat{\mathcal{G}}$ can be expressed as the solution of the $\operatorname{VI}(F, \mathcal{X} \times \Delta)$ [39, Prop. 1.4.2]. A link with the equilibria of $\widetilde{\mathcal{G}}$ is formalised next:

Proposition 8: Let Assumption 1 hold, and $z^{*}=\left(x^{*}, y^{*}\right)$ be a solution of the $\operatorname{VI}(F, \mathcal{X} \times \Delta)$. Then

(i) $x^{*}$ is a $\mathrm{NE}$ of $\widetilde{\mathcal{G}}$;

(ii) The set $\Omega$ is nonempty.

Proof: (i): By [39, Prop. 1.4.2], $z^{*}=\left(x^{*}, y^{*}\right)$ is a solution of $\widehat{\mathcal{G}}$ if and only if it solves the $\operatorname{VI}(F, \mathcal{X} \times \Delta)$; then the statement follows readily from Lemma $7 .^{2}$ (ii): Given Assumption 1 and the compactness of $\Delta$, the $\operatorname{VI}(F, \mathcal{X} \times \Delta)$ has at least one solution [39, Cor. 2.2.5]. Nonemptiness of $\Omega$ then follows from the previous point.

\section{B. Monotonicity of $F$}

The development of algorithms for the solution of VI problems relies upon the monotonicity of the mapping $F$ in (8), which plays a role analogous to convexity in optimization [8].

Definition 9 (Monotonicity): A mapping $F: \mathcal{D} \rightarrow \mathbb{R}^{d}$, with $\mathcal{D} \subseteq \mathbb{R}^{d}$ closed and convex, is

\footnotetext{
${ }^{2}$ Compactness of $\mathcal{X}$ in Assumption 1 is only needed for (ii), closedness is sufficient for (i).
}

- monotone on $\mathcal{D}$ if $(z-w)^{\top}(F(z)-F(w)) \geq 0$, and

- strongly monotone on $\mathcal{D}$ if there exists $c>0$ such that $(z-w)^{\top}(F(z)-F(w)) \geq c\|z-w\|^{2}$,

for all $z, w \in \mathcal{D}$.

The following result is instrumental in our discussion:

Lemma 10: Let Assumptions 1 and 2 hold. Then

(i) $F(x, y)$ in (11) is monotone on $\mathcal{X} \times \Delta$;

(ii) The game $\widehat{\mathcal{G}}$ admits multiple NEs.

Proof: (i): First, note that by Assumption $2 F(x, y)$ is continuously differentiable on its domain. Let $F^{x}$ and $F^{y}$ respectively denote the first $n$ and the last $M$ rows of $F$, i.e., $F^{x}(x, y)=\left(\nabla_{x_{i}} f_{i}(x)+\nabla_{x_{i}} g(x, y)\right)_{i \in \mathcal{N}}$, and $F^{y}(x, y)=$ $\left(\nabla_{y_{m}} g(x, y)\right)_{m=1}^{M}$. By definition of Jacobian we have

$$
\begin{array}{r}
\mathbf{J} F(x, y)=\left[\begin{array}{cc}
\mathbf{J}_{x} F^{x}(x, y) & \mathbf{J}_{y} F^{x}(x, y) \\
-\mathbf{J}_{x} F^{y}(x, y) & \mathbf{J}_{y} F^{y}(x, y)
\end{array}\right] \\
=\left[\begin{array}{cc}
\mathbf{J}_{x} F^{x}(x, y) & R \\
-R^{\top} & 0
\end{array}\right],
\end{array}
$$

where $\mathbf{J}_{x} F^{x}=\left(\nabla_{x_{i} x_{j}}^{2}\left(f_{i}(x)+\hat{g}(x, y)\right)\right)_{i, j \in \mathcal{N}}$ and $\mathbf{J}_{y} F^{x}=$ $\left(\nabla_{x_{i} y}^{2}\left(f_{i}(x)+\hat{g}(x, y)\right)\right)_{i \in \mathcal{N}}$ (similar definitions apply to the remaining terms). Assumption 1 implies the existence of $\chi^{f} \in \mathbb{R}$ and $\chi^{g} \in \mathbb{R}$ such that, for all $(x, y) \in \mathcal{X} \times \Delta$,

$$
\begin{aligned}
\nu^{\top}\left(\nabla_{x_{j} x_{i}}^{2} f_{i}(x)\right)_{i, j} \nu & \geq \chi^{f}\|\nu\|^{2}, \\
\nu^{\top} \nabla_{x x}^{2} \hat{g}(x, y) \nu & \geq \chi^{g}\|\nu\|^{2}, \forall \nu \in \mathbb{R}^{T} .
\end{aligned}
$$

with $\chi^{f}+\chi^{g} \geq 0$ for the convexity assumption to hold. Summing the above inequalities yields

$$
\begin{aligned}
& \nu^{\top}\left(\nabla_{x_{j} x_{i}}^{2} f_{i}(x)+\nabla_{x_{j} x_{i}}^{2} \hat{g}(x, y)\right)_{i, j} \nu \\
& \quad=\nu^{\top} \mathbf{J}_{x} F^{x}(x, y) \nu \geq\left(\chi^{f}+\chi^{g}\right)\|\nu\|^{2}, \forall \nu \in \mathbb{R}^{T}
\end{aligned}
$$

which corresponds to $\mathbf{J}_{x} F^{x}(x, y) \succeq 0$ and in turn, from (12), implies $\nu^{\top} \mathbf{J} F(x, y) \nu \geq 0$ for all $\nu \in \mathbb{R}^{n+M}$. The statement then follows directly from [39, Prop. 2.3.2].

(ii): By [8, Thm. 41] the monotonicity of $F$ implies the $\operatorname{VI}(F, \mathcal{X} \times \Delta)$ admits multiple solutions: this together with [39, Prop. 1.4.2]—-stating the correspondence between the solutions of the $\operatorname{VI}(F, \mathcal{X} \times \Delta)$ and the NEs of $\widehat{\mathcal{G}}$ - concludes the proof.

\section{Decentralized algorithm for monotone VI and equilibrium selection}

There are two main challenges: firstly, due to the possible presence of multiple equilibria, standard decentralized algorithms for VIs are not guaranteed to converge on monotone problems; a tighter condition, namely strong monotonicity, is required on the VI mapping $F$. Secondly, given the previous point is addressed, a tie-break rule needs to be put in place to select a unique solution in the presence of multiple NEs and fulfil the single-valued character of $\widetilde{\Phi}$ as required by Theorem 6.

A solution to the first issue comes from [41], [8]: these results show that proximal algorithms can be employed to retrieve $a$ solution of a monotone VI by solving a particular sequence of strongly monotone problems, derived by regularizing the original problem. We wish to emphasize that despite a single solution is obtained through these techniques, a deterministic behaviour of the associated iterative algorithm 
cannot be ensured. ${ }^{3}$ In other words, the aforementioned algorithms still correspond to multi-valued mappings. Interestingly, the work of [8] provides a workaround for this, hence allowing to address the second issue. In particular, [8, Algorithm 4] allows to solve, as a specific case,

$$
\begin{aligned}
& \text { find } z^{*}=\underset{z^{*} \in \mathcal{X} \times \Delta}{\arg \min } \frac{1}{2}\left\|z^{*}\right\|^{2} \\
& \text { s.t. }\left(z-z^{*}\right)^{\top} F\left(z^{*}\right) \geq 0, \forall z \in \mathcal{D},
\end{aligned}
$$

where $F$ is monotone. Thus, the minimum-norm equilibrium of the game $\widehat{\mathcal{G}}$ can be specified as the limit point of the algorithm, thus recovering a suitable formulation for the single-valued mapping $\widetilde{\Phi}$.

Therefore, we proceed by considering the regularized game $\widehat{\mathcal{G}}^{\tau, \bar{z}}$ where $\tau$ and $\bar{z}=(\bar{x}, \bar{y})$ are the designated step size and centre of regularization, respectively. Then, given the tuple $\left\{x_{-i}, y, \bar{x}_{i}\right\}$, each player $i \in \mathcal{N}$ solves the following problem

$$
\begin{aligned}
x^{*}=\underset{x_{i} \in \mathcal{X}_{i}}{\arg \min } & f_{i}\left(x_{i}, x_{-i}\right)+\hat{g}\left(x_{i}, x_{-i}, y\right) \\
& +\frac{\eta}{2}\left\|\left(x_{i}, x_{-i}, y\right)\right\|^{2}+\frac{\tau}{2}\left\|x_{i}-\bar{x}_{i}\right\|^{2},
\end{aligned}
$$

while the coordinator (player $N+1$ ), given $\{x, \bar{y}\}$, solves

$$
y^{*}=\underset{y \in \Delta}{\arg \max } \hat{g}(x, y)-\frac{\eta}{2}\|(x, y)\|^{2}-\frac{\tau}{2}\|y-\bar{y}\|^{2},
$$

with $\eta, \tau \in \mathbb{R}_{+}$. Note that Assumption 1 still holds for (17)(18). By taking the pseudo-gradient of the above as in (11), we have from [39, Prop. 1.4.2] that $z^{*}=\left(x^{*}, y^{*}\right)$ is a NE of $\widehat{\mathcal{G}}^{\tau, \bar{z}}$ if and only if it satisfies the VI

$$
\left(z-z^{*}\right)^{\top}\left(F\left(z^{*}\right)+\eta z^{*}+\tau\left(z^{*}-\bar{z}\right)\right) \geq 0, \forall z, \bar{z} \in \mathcal{X} \times \Delta .
$$

The next lemma is key to the use of decentralized algorithms for strongly monotone VIs on our case.

Lemma 11: Let Assumptions 1 and 2 hold; let $F$ be defined as in (11), and $\eta \geq 0$ given. Then, for any $\tau>0$ and $\bar{z} \in \mathcal{X} \times \Delta$, the regularized game $\widehat{\mathcal{G}}^{\tau, \bar{z}}$ defined by (17)-(18) has a unique NE.

Proof: Let $F^{\tau, \bar{z}}(z) \triangleq F(z)+\eta z+\tau(z-\bar{z})$. We note that $\mathbf{J} F^{\tau, \bar{z}} \succeq \tau I$ since, for all $\nu \neq 0$,

$\nu^{\top} \mathbf{J} F^{\tau, \bar{z}} \nu=\nu^{\top}(\mathbf{J} F+(\eta+\tau) I) \nu \geq \nu^{\top} \mathbf{J} F \nu+\tau \nu^{\top} \nu \geq \tau\|\nu\|^{2}$

where the last inequality follows from Lemma 10. By definition of convexity this implies $(z-w)^{\top}\left(F^{\tau, \bar{z}}(z)-F^{\tau, \bar{z}}(w)\right) \geq$ $\tau\|z-w\|^{2}$ for all $z, w \in \mathcal{X} \times \Delta$, corresponding to the definition of strongly monotone mapping. The statement is then implied by [8, Thm. 41].

Now let $S^{\tau}(\cdot)$ denote the solution of the $\operatorname{VI}\left(F^{\tau, \cdot}, \mathcal{X} \times \Delta\right)$. Building on Lemma 11 , the idea is to achieve a NE of $\widetilde{\mathcal{G}}$ by updating the centre of regularization of $\widehat{\mathcal{G}}^{\tau, \cdot}$ on the basis of an iterative method in the form $\bar{z}^{(k+1)}=S^{\tau}\left(\bar{z}^{(k)}\right)$, until convergence to the fixed point $z^{*}=S^{\tau}\left(z^{*}\right)$ corresponding to the NE of $\widehat{\mathcal{G}}^{\tau, z^{*}}$ satisfying (16). Algorithm 1 allows to establish such a connection, formalised in Lemma 12.

Lemma 12 (Thm. 21 [8]): Consider the minmax game $\widetilde{\mathcal{G}}$ defined by (9)-(10) and the regularized augmented game

\footnotetext{
${ }^{3} \mathrm{~A}$ deeper discussion on this point is out of the scope of this paper.
}

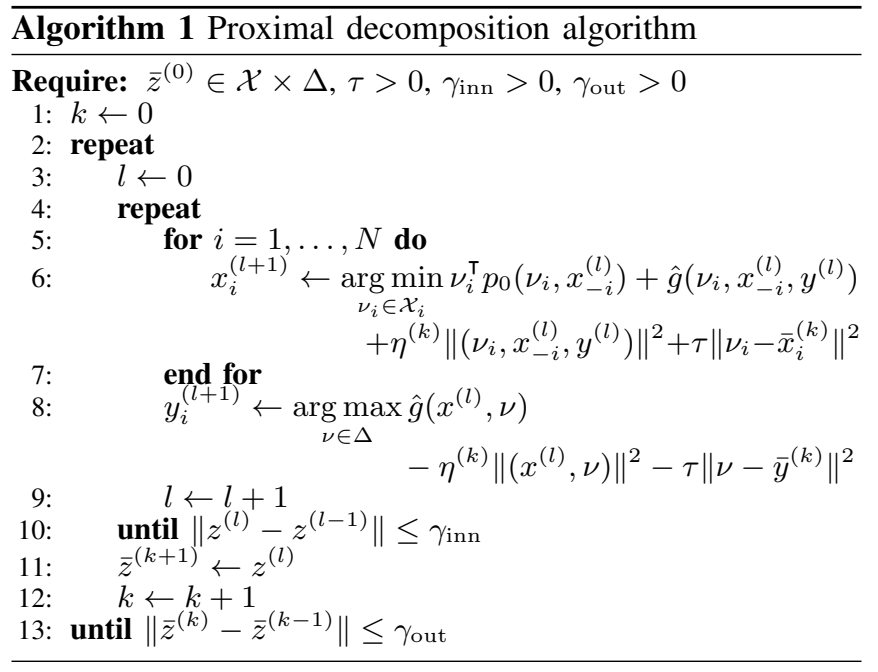

$\widehat{\mathcal{G}}^{\tau, \cdot}$ defined by (17)-(18), and let Assumptions 1 and 2 hold. Let $\left\{\eta^{(k)}\right\}_{k=0}^{\infty}$ be any sequence satisfying $\eta^{(k)}>0$ for all $k, \sum_{k=0}^{\infty} \eta^{(k)}=\infty$, and $\lim _{k \rightarrow \infty} \eta^{(k)}=0$. Let $\bar{\tau}>0$ be such that with $\tau=\bar{\tau}$ steps $3-11$ of Algorithm 1 constitute a block contraction [42], and let $\left\{\bar{z}^{(k)}\right\}_{k=0}^{\infty}$ denote the sequence generated by the Algorithm. For any $\tau \geq \bar{\tau}$, there exists $\gamma_{\mathrm{inn}}, \gamma_{\text {out }}>0$ such that $\left\{\bar{z}^{(k)}\right\}_{k=0}^{\infty}$ is bounded, and $z^{*}=\left(x^{*}, y^{*}\right)$ solution of (16) such that $\left\|\bar{z}^{(k)}-z^{*}\right\| \rightarrow 0$ for $k \rightarrow \infty$. Moreover, there exists $x \in \Omega$ such that $x=x^{*}$.

Proof: By [8, Thm. 21], Algorithm 1 asymptotically converges to a solution of (16). By Proposition 8, (16b) is equivalent to the game $\widehat{\mathcal{G}}$, whose solution set is nonempty and-by Lemma 7 -contained in $\Omega$.

The main implication of Lemma 12 is to provide a practical implementation of the single-valued mapping $\widetilde{\Phi}: \Theta^{M} \rightarrow \Omega$, thus formally proving point (i) in Theorem 6.

\section{A POSTERIORI ANALYSIS}

\section{A. Proof of Theorem 6}

(i): The construction of Section IV-C provides a proof for the existence of a single-valued mapping $\widetilde{\Phi}$.

(ii): The proof relies on the existence of a single-valued mapping from $\left\{\theta_{1}, \ldots, \theta_{M}\right\}$ to the decision space [29, Remark 2], in our case verified by $\widetilde{\Phi}$, while Assumption 4 defines necessary measurability properties of the probability space that enable the use of fundamental results from statistical learning theory; see, e.g., [32] and references therein. The mapping $\widetilde{\Phi}$ returns an NE $x^{*}$ of $\widetilde{\mathcal{G}}$. Recall that, by (11) and (16), we can obtain $x^{*}$ as the first component of the solution of the following optimization program (note the slight abuse of notation as by $\left(x^{*}, y^{*}\right)$ we denote both the optimizer and the corresponding decision variables)

$$
\begin{array}{ll} 
& \min _{\left(x^{*}, y^{*}\right) \in \mathcal{X} \times \Delta}\left\|\left(x^{*}, y^{*}\right)\right\|^{2} \\
\text { s.t. } & \sum_{i \in \mathcal{N}}\left(x_{i}-x_{i}^{*}\right)^{\top} \nabla_{x_{i}}\left(f_{i}\left(x^{*}\right)+\hat{g}\left(x^{*}, y^{*}\right)\right) \\
& -\sum_{m=1}^{M}\left(y_{m}-y_{m}^{*}\right) \nabla_{y_{m}} \hat{g}\left(x^{*}, y^{*}\right) \geq 0, \forall x \in \mathcal{X}, y \in \Delta .
\end{array}
$$


Recalling $\nabla_{y_{m}}\left(\sum_{m=1}^{M} y_{m}^{*} g\left(x^{*}, \theta_{m}\right)\right)=g\left(x^{*}, \theta_{m}\right)$, and by definition of $\hat{g}$ in (9), (21b) can be equivalently written as

$$
\begin{aligned}
& \sum_{i \in \mathcal{N}}\left(x_{i}-x_{i}^{*}\right)^{\top} \nabla_{x_{i}}\left(f_{i}\left(x^{*}\right)+\sum_{m=1}^{M} y_{m}^{*} g\left(x^{*}, \theta_{m}\right)\right) \\
&+\sum_{m=1}^{M} y_{m}^{*} g\left(x^{*}, \theta_{m}\right)-\sum_{m=1}^{M} y_{m} g\left(x^{*}, \theta_{m}\right) \geq 0, \\
& \forall x \in \mathcal{X}, y \in \Delta .
\end{aligned}
$$

As (22) holds for all $y \in \Delta$, we have that (21b) is equivalent to the following inequality being satisfied for all $x \in \mathcal{X}$,

$$
\begin{aligned}
& \sum_{i \in \mathcal{N}}\left(x_{i}-x_{i}^{*}\right)^{\top} \nabla_{x_{i}}\left(f_{i}\left(x^{*}\right)+\sum_{m=1}^{M} y_{m}^{*} g\left(x^{*}, \theta_{m}\right)\right) \\
&+\sum_{m=1}^{M} y_{m}^{*} g\left(x^{*}, \theta_{m}\right)-\max _{y \in \Delta} \sum_{m=1}^{M} y_{m} g\left(x^{*}, \theta_{m}\right) \\
&=\sum_{i \in \mathcal{N}}\left(x_{i}-x_{i}^{*}\right)^{\top} \nabla_{x_{i}}\left(f_{i}\left(x^{*}\right)+\sum_{m=1}^{M} y_{m}^{*} g\left(x^{*}, \theta_{m}\right)\right) \\
& \quad+\sum_{m=1}^{M} y_{m}^{*} g\left(x^{*}, \theta_{m}\right)-\max _{m \in\{1, \ldots, M\}} g\left(x, \theta_{m}\right) \geq 0 .
\end{aligned}
$$

Then, by replacing (16b) with (23), the resulting problem is amenable to the theory of [29], which accompanies the minimizer with probabilistic certificates on the satisfaction of its constraints. Note that (21) is in fact convex (even though this is not required by [29]), as (23) is equivalent to the VI constraint (16b), which is convex under Assumption 1 [8, $\S I V-C]$. Since the constraints in (23) are equivalent to (21b), i.e. to the definition of a NE of $\widehat{\mathcal{G}},[29, \mathrm{Thm}$. 1] entails that with confidence at least $1-\beta$, the probability that $\left(x^{*}, y^{*}\right)$ is not an equilibrium of $\widehat{\mathcal{G}}$ when a new $\theta \in \Theta$ is realized is at most $\varepsilon(\bar{n})$. By Proposition 8, the last statement is equivalent to (7), or in other words, with confidence at least $1-\beta$, $V\left(x^{*}\right)$, i.e., the probability that $x^{*} \notin \Omega^{+}$is at most $\varepsilon(\bar{n})$.

\section{B. Enumeration of support constraints}

Since the problem is convex (as derived in Section V-A) it follows from Definition 5 that the set of support samples is necessarily a subset of the active constraints. From this observation, we derive the following result, providing an efficient means of estimating the support dimension $\bar{n}$ for the considered noncooperative minmax game:

Proposition 13: Let Assumptions 1, 2 hold, and consider the function $\hat{g}: \mathcal{X} \times \Delta \rightarrow \mathbb{R}$ defined in (9). For any solution $z^{*}=\left(x^{*}, y^{*}\right)$ of the augmented game $\widehat{\mathcal{G}}$, it holds $\bar{n} \leq\left|\mathcal{Y}^{*}\right|$, where $\mathcal{Y}^{*} \triangleq\left\{m \in\{1, \ldots, M\}: y_{m}^{*}>0\right\}$.

Proof: For any fixed $\left\{\theta_{m}\right\}_{m=1}^{M}$, let us consider the following epigraphic reformulation of $\widetilde{\mathcal{G}}$

$$
\begin{aligned}
& \left(x_{i}^{*}, \gamma^{*}\right) \in \underset{x_{i}, \gamma}{\arg \min } f_{i}\left(x_{i}, x_{-i}^{*}\right)+\gamma \\
& \text { s.t. } x_{i} \in \mathcal{X}_{i} \text {, } \\
& g\left(x_{i}, x_{-i}^{*}, \theta_{m}\right) \leq \gamma, \forall m \in\{1, \cdots, M\},
\end{aligned}
$$

for all $i \in \mathcal{N}$, and let $x^{*} \in \Omega$ be a given equilibrium. Then $\gamma^{*}$ will express the maximum value-common to all agentsof $g\left(x^{*}, \cdot\right)$, achieved for some $\theta^{*} \in\left\{\theta_{1}, \cdots, \theta_{M}\right\}$. We can thus designate the active uncertainty samples by the set $\mathcal{M}^{*}\left(\theta_{1}, \ldots, \theta_{M}\right) \triangleq\left\{m \in\{1, \ldots, M\}: g\left(x^{*}, \theta_{m}\right)=\gamma^{*}\right\} ;$ the support dimension is therefore bounded as $\bar{n} \leq\left|\mathcal{M}^{*}\right|$, where $|\cdot|$ returns the cardinality of its argument. Now we observe that the optimization variable $y \in \Delta$ introduced in the augmented game $\widehat{\mathcal{G}}$ (see (9)-(10)) can be interpreted as the shadow price of the constraint (24c) in the KKT optimality conditions corresponding to problem (24) [40, $\S 6.2 .1]$. Then by complementary slackness we have

$$
y_{m}^{*}\left(g\left(x^{*}, \theta_{m}\right)-\gamma^{*}\right)=0, \forall m \in\{1, \ldots, m\},
$$

hence $\mathcal{M}^{*}=\mathcal{Y}^{*}=\left\{m \in\{1, \ldots, M\}: y_{m}^{*}>0\right\}$, and $\bar{n} \leq$ $\left|\mathcal{M}^{*}\right|=\left|\mathcal{Y}^{*}\right|$, concluding the proof.

The important implication of Proposition 13 is that an estimate of the support dimension is readily available with the solution of $\widehat{\mathcal{G}}$ computed by means of Algorithm 1 . We emphasize that the obtained estimate is exact unless $(i)$ some component of the global solution $x^{*}$ is fixed by effect of active constraints in $\mathcal{X}$, or $(i i)$ the constraints expressing the uncertainty samples are degenerate.

\section{NUMERICAL EXAMPLE: COORDINATED EV CHARGING PROBLEM REVISITED}

In the following, the strategy vector $x_{i}$ of each EV describes the charging demand over $T$ time slots. For simplicity we consider time slots of unit length $(1 \mathrm{~h})$. We suppose that the energy price in (2) is well approximated by an affine function of the aggregate strategy $\sigma(x): x \mapsto \sum_{i \in \mathcal{N}} x_{i}$. Accordingly, $p_{0}(x)=A_{0} \sigma(x)+b_{0}$, and $p_{\theta}\left(x, \theta_{m}\right)=$ $A_{m} \sigma(x)+b_{m}$, where $A_{m} \in \mathbb{R}^{T \times T}$, for $m=0, \ldots, M$, are diagonal matrices, and $b_{m} \in \mathbb{R}^{T}$. Historical price data are used to derive (e.g., through standard scenario generation techniques [31]) the set $\left\{\theta_{m}\right\}_{m=1}^{M}$ characterizing the uncertain price component $p_{\theta}$. Moreover, we assume the charging operations are subject to $\mathcal{X}_{i} \triangleq\left\{x_{i} \in \mathbb{R}^{T}: \mathbf{1}^{\top} x_{i}=E_{i}, 0 \leq\right.$ $\left.\left\|x_{i}\right\|_{\infty} \leq P_{i}\right\}$, where $E_{i}, P_{i} \in \mathbb{R}$ respectively designate the desired final state of charge (SoC) and the maximum power deliverable by the charger.

We analyse the results of several randomly generated cases, differing in the parameters characterizing the EV constraints $\mathcal{X}_{i}$, selected from a uniform random distribution: specifically, $P_{i} \in[6,15] \mathrm{kW}$, and $E_{i}$ is chosen to be feasible in the specified time interval ( $\sim 0-35 \mathrm{kWh}$ per $12 \mathrm{~h}$ interval). Regarding the uncertainty samples, the pairs $\left\{A_{m}, b_{m}\right\}_{m=1}^{M}$ are i.i.d. extracted from $(i)$ a lognormal distribution for the diagonal entries of $A_{m}$, and $(i i)$ a positive-valued uniform distribution for the vectors $b_{m}$. The nominal electricity price, i.e., the diagonal entries $\left\{a_{t}\right\}_{t=1}^{T}$ of the matrix $A_{0}$, have been derived by rescaling a winter weekday demand profile in the UK [43], whereas $b_{0}=0$. The robust charging schedules have been obtained by implementing Algorithm 1 with $\gamma_{\text {inn }}=10^{-14}, \gamma_{\text {out }}=10^{-5}$ and $\tau \in[4,6]$; the setting considered in this example can be cast into a decentralized QP optimization, which has been efficiently solved on a dualcore 7th gen. Intel processor using Matlab.

Table I shows the mean robustness performance of several solutions (with $N=20, T=24$ ), obtained from different sets of $M=500$ samples, grouped by the resulting support dimension $\bar{n}$. The violation rate $V\left(x^{*}\right)$ of each solution is recorded over $10^{6}$ newly extracted samples (according to the same aforementioned distributions). Consistently with [29], we observe that the support dimension is indicative 
TABLE I

VIOLATION RATE

\begin{tabular}{rcccc}
\hline $\bar{n}$ & 4 & 6 & 7 & 9 \\
Empirical rate [\%] & 0.98 & 1.09 & 1.26 & 1.33 \\
Theorem 6 bound [\%] & 8.06 & 9.76 & 10.55 & 12.06 \\
\hline
\end{tabular}

of the confidence level on the equilibrium robustness. The experimental results are compared with the theoretical bound provided by Theorem 6 . In this case, the conservativeness of the latter can be addressed to the relatively small number of samples (500) employed for the computation of the NE. We observe that a much tighter bound $(\sim 2-3 \%)$ can be achieved in the same case by increasing the size of the sample to $M=2000$; this naturally comes at the price of an increase of the computational requirements, which is nonetheless (practically) independent of the number of agents, due to the decentralized scheme employed.

A visual representation of the concept of support constraint is given in Fig. 1. The plot depicts the curves expressing the uncertain cost term $N g\left(x^{*}, \theta_{m}\right)$ associated to a subset $m \in\{53,72,282,566\}$ of the $M=1000$ samples used for the derivation of the $\mathrm{NE} x^{*}$. Values are plotted as a function of the aggregate demand $\left(\left.\sigma(x)\right|_{t=1},\left.\sigma(x)\right|_{t=2}\right)$ on an interval around $\sigma\left(x^{*}\right)$. In this case the support dimension $\bar{n}=2$, with $\left\{\theta_{53}, \theta_{282}\right\}$ supporting the solution together with the constraint on the target SoC. This is shown by the plane in transparent blue, representing all possible values of the aggregate strategies satisfying $\sum_{i \in \mathcal{N}} E_{i}$ over the considered time interval. Note that in this case the constraints on the power rate $P_{i}$ are not active, and omitted from the plot for clarity.

We observed over several trials that this setting reveals some structure with respect to the considered uncertainty. In particular, a specific relation between $\bar{n}$ and the dimension of the individual decision variable $T$ holds in place. As shown in Fig. 2, experimental results suggest that $\bar{n}$ is bounded by $T$. Interestingly, at the same time we observed no sensitivity to the variation of the number of agents $N$.

Finally, Figs. 3-4 show the convergence of Algorithm 1 in the computation of the NE for $N=20 \mathrm{EVs}, T=24$ and $M=500$. A dominant linear convergence rate can be observed, and less than 4000 outer loop iterations were needed to meet the desired exit accuracy $\gamma_{\text {out }}$. The inner loop enjoys similar convergence rate (not shown for space reasons), and less than 30 iterations (15 in average) are needed to achieve an error smaller than $\gamma_{\text {inn }}=10^{-14}$.

\section{CONCLUSION}

We considered multi-agent games in the presence of uncertainty. We robustified agents' strategies against unknown externalities acting on the objective function by defining a suitable noncooperative minmax game. In doing this, we adopted a data driven paradigm where uncertainty is represented by means of scenarios. Building on results from [29], we showed how the NEs of the minmax game can be accompanied with robust sensitivity certificate, providing confidence on the probability that the computed strategies remain unaffected when a new uncertainty realisation is encountered. Nonetheless, experimental results suggest that tighter bounds could be achieved, at least in the considered case. This point is being addressed in more detail by ongoing work from the authors, specifically focusing on the structure of the addressed problem with respect to the uncertainty [36].

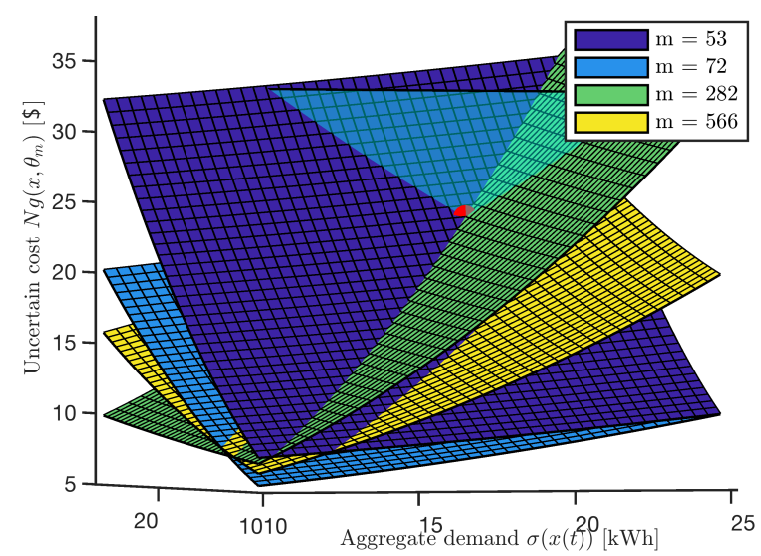

Fig. 1. Case with $N=20, T=2$ : Uncertain cost component $N g\left(x^{*}, \theta_{m}\right)$ for a subset $m \in\{53,72,282,566\}$ of the $M=1000$ samples used for the derivation of the NE $x^{*}$. Curves are plotted as a function of the aggregate demand $\left(\left.\sigma(x)\right|_{t=1},\left.\sigma(x)\right|_{t=2}\right)$ on an interval around $\sigma\left(x^{*}\right)$, plotted in red. In this case $\bar{n}=2$, with $\left\{\theta_{53}, \theta_{282}\right\}$ supporting the solution together with the SoC constraint. This is shown by the plane in transparent blue, representing all aggregate strategies fulfilling $\left.\sigma(x)\right|_{t=1}+\left.\sigma(x)\right|_{t=2}=$ $\sum_{i \in \mathcal{N}} E_{i}$ over the considered time interval. Constraints on the power rate $P_{i}$ are not active, and omitted for clarity.

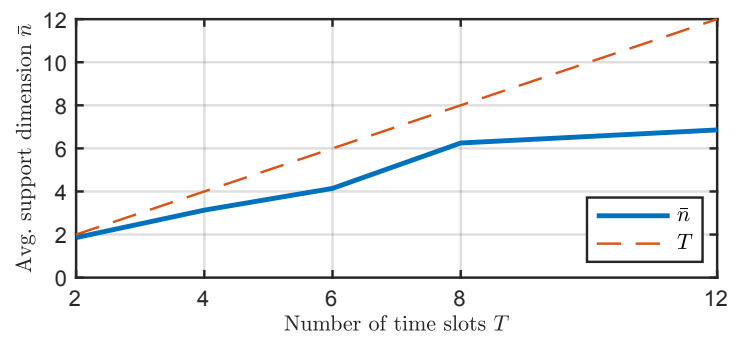

Fig. 2. Sensitivity of $\bar{n}$ to the dimension of the individual decision variable $T$. The plot shows the average support dimension $\bar{n}$ resulting over 50 trials, corresponding to different randomly generated cases, for different values of $T$ and $M$. In all cases, $\bar{n}$ is bounded by $T$. Also, we observed no sensitivity to the variation of the number of agents $N$.

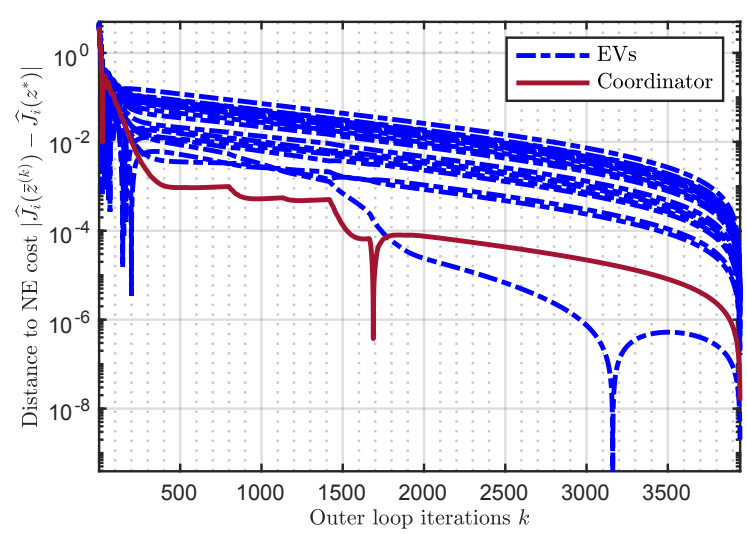

Fig. 3. Convergence of EV agents' and coordinator's costs (outer loop), for $N=20, T=24, M=500$ : a dominant linear convergence rate can be observed. With a slight abuse of notation, $\widehat{J}_{i}$ denotes the cost of each player in the augmented game $\widehat{\mathcal{G}}$ (i.e., $i=1, \ldots, N+1$ ), defined as the objective functions in (9)-(10). 


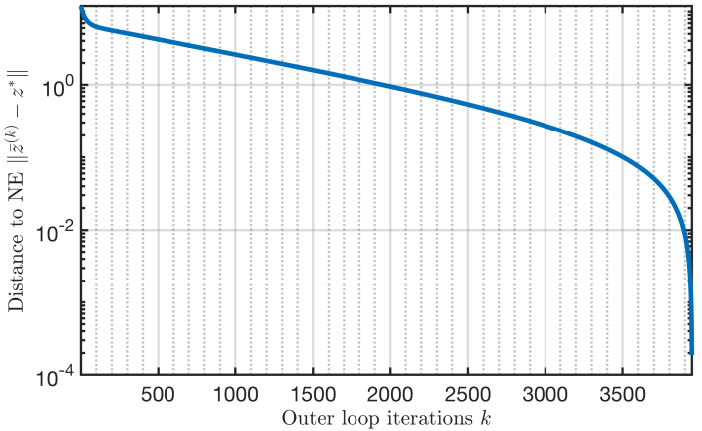

Fig. 4. Convergence of the solution vector to the NE (outer loop) for $N=20, T=24, M=500$ : a dominant linear convergence rate can be observed.

Future work will also evaluate the possibility of using the readily available shadow prices associated to the support samples as a tuning parameter to achieve the desired tradeoff between robustness and performance [38], [44].

\section{REFERENCES}

[1] F. Lamnabhi-Lagarrigue, A. Annaswamy, S. Engell, A. Isaksson, P. Khargonekar, R. M. Murray, H. Nijmeijer, T. Samad, D. Tilbury, and P. V. den Hof, "Systems \& control for the future of humanity, research agenda: Current and future roles, impact and grand challenges," Annual Reviews in Control, vol. 43, pp. 1 - 64, 2017.

[2] T. Basar and G. Olsder, Dynamic Noncooperative Game Theory, 2nd Edition. Society for Industrial and Applied Mathematics, 1998.

[3] M. Aghassi and D. Bertsimas, "Robust game theory," Mathematical Programming, vol. 107, no. 1, pp. 231-273, Jun 2006.

[4] F. Facchinei and C. Kanzow, "Generalized Nash equilibrium problems," 4OR, vol. 5, no. 3, pp. 173-210, Sep 2007.

[5] A. Mohsenian-Rad, V. W. S. Wong, J. Jatskevich, R. Schober, and A. Leon-Garcia, "Autonomous demand-side management based on game-theoretic energy consumption scheduling for the future smart grid," IEEE Transactions on Smart Grid, vol. 1, no. 3, pp. 320-331, Dec 2010.

[6] A. De Paola, F. Fele, D. Angeli, and G. Strbac, "Distributed coordination of price-responsive electric loads: A receding horizon approach," in 2018 IEEE Conference on Decision and Control (CDC), Dec 2018, pp. 6033-6040.

[7] D. Paccagnan, B. Gentile, F. Parise, M. Kamgarpour, and J. Lygeros, "Nash and Wardrop equilibria in aggregative games with coupling constraints," IEEE Transactions on Automatic Control, pp. 1-1, 2018.

[8] G. Scutari, F. Facchinei, J. S. Pang, and D. P. Palomar, "Real and complex monotone communication games," IEEE Transactions on Information Theory, vol. 60, no. 7, pp. 4197-4231, July 2014.

[9] I. Atzeni, L. G. Ordóñez, G. Scutari, D. P. Palomar, and J. R. Fonollosa, "Noncooperative day-ahead bidding strategies for demandside expected cost minimization with real-time adjustments: A GNEP approach," IEEE Transactions on Signal Processing, vol. 62, no. 9, pp. 2397-2412, May 2014.

[10] J. G. Wardrop, "Some theoretical aspects of road traffic research." Proceedings of the Institution of Civil Engineers, vol. 1, no. 3, pp. 325-362, 1952.

[11] S. Grammatico, "Dynamic control of agents playing aggregative games with coupling constraints," IEEE Transactions on Automatic Control, vol. 62, no. 9, pp. 4537-4548, Sep. 2017.

[12] L. Deori, K. Margellos, and M. Prandini, "Price of anarchy in electric vehicle charging control games: When Nash equilibria achieve social welfare," Automatica, vol. 96, pp. $150-158,2018$.

[13] D. Paccagnan, F. Parise, and J. Lygeros, "On the efficiency of Nash equilibria in aggregative charging games," IEEE Control Systems Letters, vol. 2, no. 4, pp. 629-634, Oct 2018.

[14] F. Fele, A. De Paola, D. Angeli, and G. Strbac, "A framework for receding-horizon control in infinite-horizon aggregative games," Annual Reviews in Control, vol. 45, pp. 191 - 204, 2018.

[15] J. Nash, "Non-cooperative games," Annals of Mathematics, vol. 54, no. 2, pp. 286-295, 1951.

[16] J.-S. Pang, S. Sen, and U. V. Shanbhag, "Two-stage non-cooperative games with risk-averse players," Mathematical Programming, vol. 165, no. 1, pp. 235-290, Sep 2017.

[17] S. K. Khaitan and J. D. McCalley, "Design techniques and applications of cyberphysical systems: A survey," IEEE Systems Journal, vol. 9, no. 2, pp. 350-365, June 2015.
[18] K. Kim and P. R. Kumar, "Cyber-physical systems: A perspective at the centennial," Proceedings of the IEEE, vol. 100, no. Special Centennial Issue, pp. 1287-1308, May 2012.

[19] J. C. Harsanyi, "Games with incomplete information played by "bayesian" players, i-iii part i. the basic model," Management Science, vol. 14 , no. 3 , pp. $159-182,1967$.

[20] P. Couchman, B. Kouvaritakis, M. Cannon, and F. Prashad, "Gaming strategy for electric power with random demand," IEEE Transactions on Power Systems, vol. 20, no. 3, pp. 1283-1292, 2005.

[21] V. V. Singh, O. Jouini, and A. Lisser, "Existence of nash equilibrium for chance-constrained games," Operations Research Letters, vol. 44 no. 5 , pp. $640-644,2016$.

[22] U. Ravat and U. Shanbhag, "On the characterization of solution sets of smooth and nonsmooth convex stochastic nash games," SIAM Journal on Optimization, vol. 21, no. 3, pp. 1168-1199, 2011.

[23] J. Koshal, A. Nedic, and U. V. Shanbhag, "Regularized iterative stochastic approximation methods for stochastic variationalinequality problems," IEEE Transactions on Automatic Control, vol. 58, no. 3, pp. 594-609, March 2013.

[24] H. Xu and D. Zhang, "Stochastic nash equilibrium problems: sample average approximation and applications," Computational Optimization and Applications, vol. 55, no. 3, pp. 597-645, Jul 2013.

[25] C. Yu, M. van der Schaar, and A. H. Sayed, "Distributed learning for stochastic generalized nash equilibrium problems," IEEE Transactions on Signal Processing, vol. 65, no. 15, pp. 3893-3908, Aug 2017.

[26] J. Lei and U. V. Shanbhag, "A randomized inexact proximal bestresponse scheme for potential stochastic nash games," in 2017 IEEE 56th Annual Conference on Decision and Control (CDC), Dec 2017, pp. 1646-1651.

[27] R. Nishimura, S. Hayashi, and M. Fukushima, "Robust Nash equilibria in $n$-person non-cooperative games: Uniqueness and reformulation," Pacific J. Optim., no. 5, 2009.

[28] M. Hu and M. Fukushima, "Existence, uniqueness, and computation of robust Nash equilibria in a class of multi-leader-follower games," SIAM Journal on Optimization, vol. 23, no. 2, pp. 894-916, 2013.

[29] M. C. Campi, S. Garatti, and F. A. Ramponi, "A general scenario theory for non-convex optimization and decision making," IEEE Transactions on Automatic Control, pp. 1-1, 2018.

[30] G. C. Calafiore and M. C. Campi, "The scenario approach to robust control design," IEEE Transactions on Automatic Control, vol. 51, no. 5, pp. 742-753, May 2006.

[31] A. J. Conejo, M. Carrión, and J. M. Morales, Uncertainty Characterization via Scenarios. Boston, MA: Springer US, 2010, pp. 63-119.

[32] T. Alamo, R. Tempo, and E. F. Camacho, "Randomized strategies for probabilistic solutions of uncertain feasibility and optimization problems," IEEE Transactions on Automatic Control, vol. 54, no. 11, pp. 2545-2559, Nov 2009.

[33] M. Campi and S. Garatti, "The exact feasibility of randomized solutions of uncertain convex programs," SIAM Journal on Optimization, vol. 19, no. 3, pp. 1211-1230, 2008

[34] J. Zazo, S. Zazo, and S. V. Macua, "Robust worst-case analysis of demand-side management in smart grids," IEEE Transactions on Smart Grid, vol. 8, no. 2, pp. 662-673, March 2017.

[35] F. Facchinei, J.-S. Pang, and G. Scutari, "Non-cooperative games with minmax objectives," Computational Optimization and Applications, vol. 59, no. 1, pp. 85-112, Oct 2014.

[36] G. Schildbach, L. Fagiano, and M. Morari, "Randomized solutions to convex programs with multiple chance constraints," SIAM Journal on Optimization, vol. 23, no. 4, pp. 2479-2501, 2013.

[37] M. C. Campi and S. Garatti, "Wait-and-judge scenario optimization," Mathematical Programming, vol. 167, no. 1, pp. 155-189, Jan 2018.

[38] G. Calafiore, "Random convex programs," SIAM Journal on Optimization, vol. 20, no. 6, pp. 3427-3464, 2010.

[39] F. Facchinei and J.-S. Pang, Finite-Dimensional Variational Inequalities and Complementarity Problems. Springer-Verlag New York, 2003

[40] B. Rustem and M. Howe, Algorithms for Worst-Case Design and Applications to Risk Management. Princeton University Press, 2002.

[41] A. Kannan and U. Shanbhag, "Distributed computation of equilibria in monotone Nash games via iterative regularization techniques," SIAM Journal on Optimization, vol. 22, no. 4, pp. 1177-1205, 2012.

[42] D. P. Bertsekas and J. N. Tsitsiklis, Parallel and Distributed Computation: Numerical Methods. Upper Saddle River, NJ, USA: PrenticeHall, Inc., 1989.

[43] National Grid. (2019, Mar.) Historical demand data. [Online]. Available: https://www.nationalgrideso.com/balancing-data/data-explorer

[44] M. C. Campi and S. Garatti, "A sampling-and-discarding approach to chance-constrained optimization: Feasibility and optimality," Journal of Optimization Theory and Applications, vol. 148, no. 2, pp. 257-280, Feb 2011. 